\title{
The Case for a Return to Enceladus
}

A Science White Paper for the Planetary Science Decadal Survey

\section{Authors and Affiliations:}

Morgan L. Cable, NASA Jet Propulsion Laboratory, California Institute of Technology

Shannon MacKenzie, Johns Hopkins University/Applied Physics Laboratory

Marc Neveu, NASA Goddard Space Flight Center/University of Maryland

Tori M. Hoehler, NASA Ames Research Center

Amanda R. Hendrix, Planetary Science Institute

Jennifer Eigenbrode, NASA Goddard Space Flight Center

Frank Postberg, Freie Universitaet Berlin

Carolyn Porco, Space Sciences Lab, University of California, Berkeley CA

Christopher R. Glein, Southwest Research Institute

Linda Spilker, NASA Jet Propulsion Laboratory, California Institute of Technology

Amy E. Hofmann, NASA Jet Propulsion Laboratory, California Institute of Technology

Alfred McEwen, LPL, University of Arizona

J. Hunter Waite, Southwest Research Institute

Peter Wurz, Physics Institute, University of Bern, Switzerland

Jörn Helbert, German Aerospace Center (DLR)

Ariel Anbar, Arizona State University

Jean-Pierre de Vera, German Aerospace Center (DLR)

Jorge Núñez, Johns Hopkins University Applied Physics Laboratory

Primary Author Contact: Morgan.L.Cable@jpl.nasa.gov

\section{Endorsers:}

For a full list of endorsers, please see: https://docs.google.com/document/d/1HPQAj1hZhoXGna7iut1h8zw_jhbszFA7FRwUOQ7sM4/edit?usp=sharing

A portion of this research was carried out at the Jet Propulsion Laboratory, California Institute of Technology, under a contract with the National Aeronautics and Space Administration (80NM0018D0004). (C) 2020. All rights reserved. 


\section{Summary}

The plume of Enceladus provides access to fresh material from a habitable, subsurface ocean. The Cassini Mission, although not specifically designed for the task, was able to take advantage of the plume to conduct the best (to date) characterization of an extraterrestrial ocean. Cassini found evidence for a global, subsurface ocean rich in salts and organics, with water-rock interactions at the seafloor. Meeting the criteria of "extended regions of liquid water, conditions favorable for the assembly of complex organic molecules, and energy source(s) to sustain metabolism" (Des Marais et al., 2008), the ocean of Enceladus is habitable. The next step is to investigate whether it is inhabited. Here, we summarize the evidence for Enceladus' ocean and its habitability, identify constraints and outstanding questions on the detectability of life within Enceladus, and recommend a return to Enceladus beginning in the coming decade.

\section{An Accessible, Global Ocean}

Enceladus is a relatively small (505 km diameter) moon of Saturn, and one of the brightest objects in the solar system, suggesting that its surface is young (see Schenk et al., 2018 and references therein). It is located within Saturn's E ring, which we now understand to be fed by ice grains emanating from Enceladus' plume (Kempf et al., 2018). While Voyager data suggested a link between Enceladus and the E ring, the Cassini Mission provided the first direct evidence for the existence of the plume and is therefore credited with its discovery (Porco et al., 2006; Dougherty et al., 2006; Hansen et al., 2006; Spahn et al., 2006; Tokar et al., 2006; Waite et al., 2006). The plume is formed, in part, by 100 jets erupting from four main surface fissures, the 'Tiger Stripes', in the South Polar Terrain; material is also likely erupting from these fissures in the form of sheets (Porco et al. 2014). The presence of sodium salts in the ice grains ejected in the plume indicates that this material originates from Enceladus' ocean (Postberg et al., 2009). Modeling from Cassini data suggests that the plume is geophysically controlled (Běhounková et al., 2015; Patthoff and Kattenhorn, 2011), and therefore a long-lived phenomenon (Hemingway et al., 2020; Lainey et al., 2020).

Two independent lines of evidence show that the plume's subsurface water reservoir is not a regional sea but a global ocean. First, analysis of gravity measurements (McKinnon, 2015; Čadek et al., 2016; Beuthe et al., 2016) indicate that Enceladus is not in hydrostatic equilibrium, and that it requires isostatic compensation to fit the gravity field and excess topography. The simplest explanation for this is a floating ice shell of variable thickness atop a global ocean. Second, comparison of surface images collected over 7 years of Cassini observations imply that Enceladus' rotation has a forced physical libration (wobble) too large to be consistent with a crust grounded to the rocky core (Thomas et al., 2016). The simplest explanation is a freefloating crust, which is only possible if there is a global ocean.

The implication of a global ocean as opposed to a regional sea is important for life. A regional sea could have been formed by an impact and might only be transient, whereas a global ocean is more likely to be long-lived. This may mean that suitable conditions in the subsurface ocean of Enceladus have persisted long enough for life to gain a foothold.

Enceladus may be the only place in the solar system where we can easily sample fresh material from a global subsurface ocean without the need to dig or drill. 


\section{Hydrothermal Activity and Chemical Energy for Metabolic Processes}

Life as we know it requires liquid water and energy as either light or redox disequilibrium. While light energy is not available in the subsurface oceans of Enceladus or any of the icy moons, Enceladus' plume composition is consistent with hydrothermal activity that could sustain redox disequilibrium over long durations. On Earth, hydrothermal activity supports chemosynthetic microbial communities and is proposed by some to have enabled the origin of life (Martin et al., 2008). Three key discoveries strongly support the occurrence of hydrothermal processes at the ocean-core interface of Enceladus. The first is that the Cassini Cosmic Dust Analyzer (CDA) found nanometer-scale dust particles consisting of silica $\left(\mathrm{SiO}_{2}\right)(\mathrm{Hsu}$ et al. 2015). Particles of this specific composition and limited size range (2-8 $\mathrm{nm}$ radius) are most likely to have been formed from ongoing high-temperature $\left(>90^{\circ} \mathrm{C}\right)$ hydrothermal leaching of silicate minerals. Once formed, the nanoparticles are transported through thermal-induced convection streams from the seafloor to the near-surface plume sources, where they are incorporated into ejected ice grains and subsequently released by sputter erosion in Saturn's E ring. A second line of evidence for hydrothermal activity comes from Cassini Ion and Neutral Mass Spectrometer (INMS) plume gas measurements. The relative proportions of measured volatiles are unlikely without a gas input, as methane would be preferentially trapped in clathrate hydrates (water-ice cages) and therefore depleted in the plume (Bouquet et al. 2015). One plausible explanation is hydrothermal activity (or microbes supported by such activity), which could generate sufficient methane to match observed levels. The third piece of evidence is identification by INMS of molecular hydrogen $\left(\mathrm{H}_{2}\right)$ in the plume in a 'deep dive' flyby (E21) at an altitude of only $48 \mathrm{~km}$ (Waite et al., 2017). Molecular hydrogen is a product of serpentinization, a reaction process between ultramafic rocks (igneous rocks with high $\mathrm{Mg}$ and Fe contents) and liquid water that occurs in hydrothermal systems. This evidence further suggests that the ocean of Enceladus is in contact with, and reacting with, the rocky core through hydrothermal processes.

The flux of hydrothermally-sourced $\mathrm{H}_{2}$ and other reduced species into a $\mathrm{CO}_{2}$-containing ocean appears to provide a redox chemical disequilibrium that, on Earth, is known to support microbial metabolism. The $\mathrm{pH}$ of the Enceladus ocean is alkaline, likely in the range of 8.5-9, based on geochemical interpretations of Cassini mass spectrometry data (Glein et al., 2020). This is consistent with long-term buffering by a seafloor rich in quartz, talc and carbonate minerals. Together with the silica-rich nanograins and detection of molecular hydrogen, these results suggest a heterogeneous rocky core featuring chemical gradients between a carbonate-rich upper layer and serpentinized interior. Alkaline hydrothermal systems on Earth such as Lost City in the Mid-Atlantic Ocean (Kelley et al. 2005) are also rich in $\mathrm{H}_{2}$, and may represent the closest analogue to fluids associated with serpentinization at Enceladus' seafloor. These hydrothermal sites on Earth host teeming ecosystems based on chemosynthetic organisms.

Cassini evidence suggests that conditions in the ocean of Enceladus are consistent with hydrothermal systems on Earth, where redox chemistry is available to support rich communities of life and perhaps may have been where life first emerged.

\section{Chemically Diverse, High Mass Organics}

Cassini's CDA and INMS instruments characterized the composition of ice grains and vapor, respectively, emanating from the plume. Intriguingly, a subset of plume particles ( 4\%) contain complex organic molecules with masses greater than $200 \mathrm{u}(\mathrm{u}=$ atomic mass units). These 
organic molecules are found in relatively high abundance (>1\% by mass) in these ice particles and are consistent with unsaturated (i.e., containing double and triple carbon-carbon and carbonnitrogen bonds) and partially aromatic (i.e., containing benzene substructures) molecules (Khawaja et al. 2019). Sources of biologically available nitrogen $\left(\mathrm{NH}_{3} / \mathrm{NH}_{4}{ }^{+}\right.$and amines) have also been found by these instruments. Importantly, analysis of CDA spectra collected at different velocities indicates that the detected organics are probably fragments of even larger organic molecules (Postberg et al., 2018); this interpretation is supported by detection of diverse, highmass organics in the vapor phase at the fastest flyby velocities $(\sim 18 \mathrm{~km} / \mathrm{s})$ (Waite et al., 2009). However, these instruments lacked the mass range and resolution to characterize these fascinating organics any further, so their sources (abiotic or biotic) are currently unknown.

The Enceladus ocean appears to support a range of organic chemistry, including high-mass compounds diverse in $\mathrm{CHON}$, that could serve as building blocks for, or be the byproducts of, life in this habitable environment.

\section{Enrichment of Organics}

Processes may be occurring in the ocean to increase the concentration of organic molecules in plume particles, which could improve the likelihood of detecting trace species (such as biomolecules). In the ocean, volatile exsolution or turbulence in the conduits leading to the surface can create bubbles that rise through the water column and could scavenge organic material, including microorganisms, by cells attaching to the bubbles' surfaces and being released as a spray when the bubbles burst at the water's upper surface (Porco et al., 2017). This process is most efficient with hydrophobic molecules and surfactants, and has been shown to increase organic and microbial concentrations by orders of magnitude on Earth (Carlucci and Williams, 1965; Blanchard and Syzdek, 1972). Bubble scrubbing may also lead to an organicrich film at the ocean surface of Enceladus, similar to the sea surface microlayer seen on Earth's oceans. On Earth, the wind-induced bursting of air bubbles at the sea surface microlayer in the polar regions generates a sub-micron sea spray aerosol containing ice particles (Burrows et al., 2014). These ice particles are similar in organic and salt content to the organic-rich plume grains detected by Cassini (Postberg et al., 2018); they are also enriched 10 to 1000-fold in organic material compared to Earth ocean concentrations. If aerosols forming at the water-ice interface at Enceladus emerge from a boundary layer of organic material, this process could concentrate hydrophobic biomarkers such as certain amino and fatty acids, if present (Cable et al., 2020).

If enrichment processes are occurring on Enceladus, the potential for detecting organic biosignatures in the plume is much higher than may be expected based on estimates of bulk concentrations in the ocean alone.

\section{Constraints and Outstanding Questions}

The age of Enceladus and its liquid water ocean are still debated; Enceladus could be only 100 Myr old (Ćuk et al., 2016), although recent evidence supports an older age (Lainey et al., 2020). Whether this influences the probability that life has taken hold there depends on how long it takes for life to emerge given suitable conditions, and this is also weakly constrained. Should life exist on Enceladus, the quality and abundance of evidence thereof will depend on the balance of factors that control the production, transport, and destruction of cells and their remnants. It has 
been argued that energy flux may be the chief limitation on the productivity of an enceledan biosphere. Existing estimates of energy flux span 5 orders of magnitude and propagate into even larger uncertainty in the possible abundance of cells and rate of production of biomolecules (Cable et al., 2020). Research to better constrain both energy fluxes and biomass-energy relations (Ray et al., 2020) would better inform the expected detection limits of biosignatures. Laboratory and field work studying the limits of life as we know it on Earth can also provide context for astrobiological investigations and challenging real-world samples to test instrument thresholds

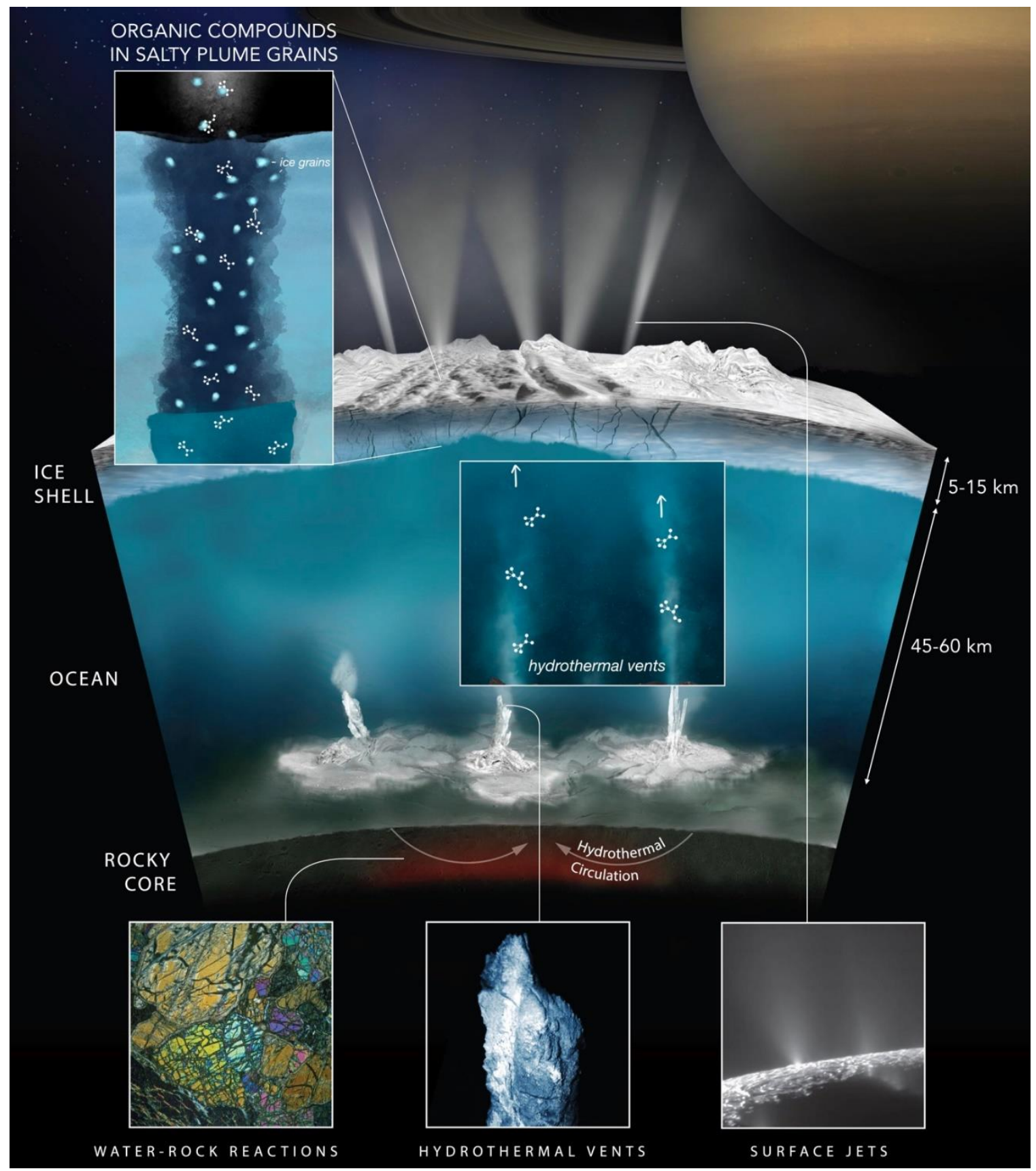

Figure 1. On Enceladus, the ingredients necessary to sustain life as we know it (liquid water, energy from water-rock reactions, bioessential elements and organic compounds) can all be sampled via the plume. So might signatures of life. Modified from PIA21442 and PIA23173 by M. Neveu. 
(e.g., Klenner et al., 2020a,b). Finally, investment in strategies and technologies to minimize the risk of contamination would help in two areas: first, reducing contamination risk for ultrasensitive life-detection payloads could lead to increased confidence that analytical results would reflect true biosignature detections; second, minimizing contamination to the ocean world environment would open to exploration regions previously declared off-limits in the interest of planetary protection, ultimately enabling more capable and wider-reaching astrobiology mission architectures.

\section{Strategies to Search for Life}

While remote sensing techniques can provide valuable information about surface composition, geology, topography, etc., ultimately in situ sampling is required to fully address the life question. Current remote sensing instruments (such as UV or NIR spectrometers) cannot unambiguously identify biomolecules (e.g., fatty acids or a biopolymer) from orbit at the subppm limits of detection expected in this potentially energy-constrained environment (Cable et al., 2020), although we note that flight instruments in development may be able to achieve ppb limits of detection by the end of the decade.

At Enceladus, in situ measurements and/or sample return could be performed via flythrough or orbital missions (by sampling the plume vapor and grains as Cassini did), or through a landed architecture. Any mission with life detection as its primary science objective should include multiple, independent tests for life, as well as thorough environmental characterization to provide context. Various payload combinations could achieve this goal (e.g., Europa Lander study, 2016; the Planetary Mission Concept Study by MacKenzie et al. entitled "The Enceladus Orbilander: A Flagship-class Mission Concept for Astrobiology"; Dachwald et al., 2020; the white paper by Neveu et al. entitled "Returning Sampling from Enceladus for Life Detection", and Tsou et al., 2012). For a detailed assessment of the geophysical context of habitability at Enceladus, see the white papers "Distributed Geophysical Exploration of Ocean Worlds" (Vance et al.) and "A recipe for geophysical exploration of Enceladus" (Ermakov et al.).

\section{Leveraging NASA Investments}

A quantitative, robust search for life beyond Earth can be carried out given our current knowledge of Enceladus and flight-ready technology. Through technology development programs like PICASSO, MatISSE, COLDTech, and ICEE-2, instruments key to life detection have reached flight maturity, including those with complementary and orthogonal biosignature measurements as well as sample ingestion, preparation, and contamination mitigation. Furthermore, NASA has invested in R\&A specifically targeting ocean worlds and life detection. These community-building efforts include establishing research coordination networks focused on themes foundational to astrobiology: developing life-detection technologies (Network for Life Detection), understanding the pathways of prebiotic chemistry (Prebiotic Chemistry and Early Earth Environments), exploring ocean worlds (Network for Ocean Worlds), and investigating the evolution of cellular life (From Early Cells to Multicellularity). Coordination between projects under these themes is well-poised to continue improving how we strategize, execute, and interpret the results of the search for life.

Thanks to NASA investments in the last decade, the available technology and community

expertise are ready to tackle one of the biggest questions in NASA's portfolio, and one that reaches beyond planetary science to impact us all: Are we alone? 


\section{Conclusions}

The Enceladus plume provides direct access to recently ejected ocean-derived materials in the plume or as surface deposits. In response to the seminal discoveries of the Cassini mission (many made after the last decadal survey), the next strategic step is to search for biosignatures in the ocean materials. This is a key objective of the 2018 NASA Strategic Plan, tackling an overarching question of the Vision \& Voyages Decadal Survey: "Beyond Earth, are there contemporary habitats [...] and do organisms live there now?". The subsurface ocean of Enceladus is the only confirmed modern environment beyond Earth with all ingredients necessary for life: liquid water, chemical building blocks, and energy sources (e.g., Des Marais et al., 2008; McKay et al., 2008, 2014; Hendrix et al., 2019; Cable et al., 2020). Current mature instrumentation and measurement strategies can enable the detection of trace quantities of key features expected to be universal to life, while also measuring the possible breadth of abiotic or prebiotic compositions if life is not present. The search for recent and extant life in Enceladus' ocean materials is possible with today's technology, and NASA investments are continuing to improve the maturity and sensitivity of these instruments. It is recommended that Enceladus receive strong consideration for New Frontiers and Flagship missions in the coming decade.

While Enceladus is not the only astrobiologically enticing icy world, the ability to sample the subsurface ocean via the plume-without the need to drill-makes Enceladus

particularly alluring and accessible as the next target for a dedicated search-for-life mission.

\section{References}

Běhounková M., G. Tobie, O. Cadek et al. (2015) Timing of water plume eruptions on Enceladus explained by interior viscosity structure. Nature Geoscience, 8, 601-606, doi: 10.1038/ngeo2475.

Beuthe M., A. Rivoldini, and A. Trinh. (2016) Enceladus's and Dione's floating ice shells supported by minimum stress isostasy. GRL, 43, 10088-10096, doi: 10.1002/2016GL070650.

Blanchard D. C. and L. D. Syzdek (1972) Concentration of bacteria in jet drops from bursting bubbles. JGR, 77, 5087-5099, doi: https://doi.org/10.1029/JC077i027p05087.

Bouquet, A., C. R. Glein, et al. (2017) Alternative energy: Production of $\mathrm{H}_{2}$ by radiolysis of water in the rocky cores of icy bodies. ApJ Lett., 840, L8, 7 pp., doi: 10.3847/2041-8213/aa6d56.

Burrows S. M., O. Ogunro, A. A. Frossard et al. (2014) A physically based framework for modeling the organic fractionation of sea spray aerosol from bubble film Langmuir equilibria. Atmos. Chem. Phys., 14, 13601-13629, doi: 10.5194/acp-14-13601-2014.

Cable, M. L., M. Neveu, H.-S. Hsu and T. Hoehler (2020) "Enceladus" in Planetary Astrobiology, Eds. V. Meadows, et al. Space Science Series. Univ. of Arizona Press and LPI. In press.

Čadek, O., G. Tobie, et al. (2016) Enceladus's internal ocean and ice shell constrained from Cassini gravity, shape, and libration data. GRL, 43, 5633-5660, doi: 10.1002/2016GL068634.

Carlucci A. F. and P. M. Williams (1965) Concentration of bacteria from seawater by bubble scavenging. CES J. Marine Sci., 30, 28-33.

Ćuk, M., L. Dones and D. Nesvorný (2016) Dynamical evidence for a late formation of Saturn's moons. The Astrophysical Journal, 820,97.

Dachwald, B., et al. (2020) Key Technologies and Instrumentation for Subsurface Exploration of Ocean Worlds. Space Sci. Rev., 216 (83), https://doi.org/10.1007/s11214-020-00707-5.

Des Marais, D. J., J. A. Nuth, et al. (2008) The NASA Astrobiology Roadmap. Astrobiology, 8, 713-730.

Dougherty M. K., K. K. Khurana, et al. (2006) Identification of a dynamic atmosphere at Enceladus with the Cassini magnetometer. Science, 311, 1406-1409, doi: 10.1126/science.1120985.

Europa Lander Study 2016 Report: Europa Lander Mission. JPL D-97667. https://solarsystem.nasa.gov/docs/Europa_Lander_SDT_Report_2016.pdf

Glein, C. R. and J. H. Waite (2020) The carbonate geochemistry of Enceladus' ocean. Geophys. Res. Lett., 47 (3), e2019GL085885. 
Hansen C. J., L. Esposito, A. I. F. Stewart et al. (2006) Enceladus' water vapor plume. Science, 311, 1422-1425, doi: $10.1126 /$ science.1121254.

Hemingway D., M. L. Rudolph, and M. Manga (2020) Cascadig parallel fractures on Enceladus. Nature Astronomy, 4, 234-239. https://doi.org/10.1038/s41550-019-0958-x.

Hendrix, A. R., T. A. Hurford, L. M. Barge, et al. (2019) The NASA Roadmap to Ocean Worlds. Astrobiology, 19, 1-27. https://doi.org/10.1089/ast.2018.1955.

Hsu, H. W., F. Postberg, et al. (2015) Ongoing hydrothermal activities within Enceladus. Nature, 519, 207-210.

Kelley, D. S., J. A. Karson, G. L. Früh-Green, et al. (2005) A serpentinite-hosted ecosystem: The Lost City Hydrothermal Field. Science, 307 (5714), 1428-1434.

Kempf, S., M. Horanyi, H. W. Hsu, T. W. Hill, et al. (2018) Saturn's diffuse E ring and its connection with Enceladus. In: Enceladus and the Icy Moons of Saturn, University of Arizona Press, pp 195-210

Khawaja, N., F. Postberg, J. Hillier, et al. (2019) Low-mass nitrogen-, oxygen-bearing, and aromatic compounds in Enceladean ice grains. Mon. Not. Royal Astronom. Soc. 489 (4), 5231-5243.

Klenner, F., et al. (2020a). Analog Experiments for the Identification of Trace Biosignatures in Ice Grains from Extraterrestrial Ocean Worlds. Astrobiology, 20 (2), 179-189. https://doi.org/10.1089/ast.2019.2065.

Klenner, F., et al. (2020b) Discriminating abiotic and biotic fingerprints of amino acids and fatty acids in ice grains relevant to ocean worlds. Astrobiology. https://doi.org/10.1089/ast.2019.2188.

Lainey, V., L. G. Casajus, J. Fuller et al. (2020) Resonance locking in giant planets indicated by the rapid orbital expansion of Titan. Nature Astronomy. https://doi.org/10.1038/s41550-020-1120-5.

Martin, W., J. Baross, D. Kelley, and M. J. Russell. (2008) Hydrothermal vents and the origin of life." Nature Reviews Microbiology, 6 (11), 805-814.

McKay, C. P. et al. (2008) The possible origin and persistence of life on Enceladus and detection of biomarkers in the plume. Astrobiology, 8, 909-19. https://doi.org/10.1089/ast.2008.0265.

McKay, C. P., A. D. Anbar, C. Porco, and P.Tsou (2014) Follow the Plume: The Habitability of Enceladus. Astrobiology, 14 (4), 352-55. https://doi.org/10.1089/ast.2014.1158.

McKinnon W. B. (2015) Effect of Enceladus's rapid synchronous spin on interpretation of Cassini gravity. GRL, 42 (7), 2137-2143.

Patthoff, D. and S. Kattenhorn (2011) A fracture history on Enceladus provides evidence for a global ocean. GRL., 38, L18201.

Porco C. C., P. Helfenstein, P. C. Thomas et al. (2006) Cassini observes the active South Pole of Enceladus. Science, 311 (5766), 1393-1401, doi: 10.1126/science.1123013.

Porco, C. C., D. DiNino et al. (2014) How the geysers, tidal stresses, and thermal emission across the South Polar Terrain of Enceladus are related. Astron. J., 148 (3), 45. https://doi.org/10.1088/0004-6256/148/3/45.

Porco, C. C., et al. (2017) Could It Be Snowing Microbes on Enceladus? Assessing Conditions in Its Plume and Implications for Future Missions. Astrobiology 17, 876-901. https://doi.org/10.1089/ast.2017.1665.

Postberg, F., S. Kempf, et al. (2009) Sodium salts in E-ring ice grains from an ocean below the surface of Enceladus. Nature 459, 1098-1101. https://doi.org/10.1038/nature08046.

Postberg, F., N. Khawaja, B. Abel et al. (2018) Macromolecular Organic Compound s from the Depths of Enceladus. Nature, 558, 564-568. https://doi.org/10.1038/s41586-018-0246-4.

Ray, C., C. R. Glein, J. H. Waite, B. Teolis, et al. (2020) Oxidation processes diversity the metabolic menu on Enceladus. Icarus, in review.

Schenk, P. M., et al. (2018) Enceladus and the Icy Moons of Saturn. $1^{\text {st }}$ ed. U. of Arizona Press: Tucson, AZ.

Spahn F., J. Schmidt, N. Albers et al. (2006) Cassini dust measurements at Enceladus and implications for the origin of the E ring. Science, 311 (5766), 1416-1418, doi: 10.1126/science.1121375.

Thomas P. C., R. Tajeddine, M. S. Tiscareno et al. (2016) Enceladus's measured physical libration requires a global subsurface ocean. Icarus, 264, 37-47, doi: 10.1016/j.icarus.2015.08.037.

Tokar R. L., R. E. Johnson, T. W. Hill et al. (2006) The interaction of the atmosphere of Enceladus with Saturn's plasma. Science, $311,1409-1412$, doi: 10.1126/science.1121061.

Tsou, P., et al. (2012) LIFE: A life investigation for Enceladus, a sample return mission concept in search for evidence of life. Astrobiology, 12 (8), 730-742. https://doi.org/10.1089/ast.2011.0813.

Waite, J. H., M. R. Combi, W.-H. Ip et al. (2006) Cassini ion and neutral mass spectrometer: Enceladus plume composition and structure. Science, 311 (5766), 1419-1422, doi: 10.1126/science.1121290.

Waite, J. H. et al. (2009) Liquid water on Enceladus from Observations of Ammonia and $40 \mathrm{Ar}$ in the plume. Nature, 460, 487-490.

Waite, J. H. et al. (2017) Cassini Finds Molecular Hydrogen in the Enceladus Plume: Evidence for Hydrothermal Processes. Science, 356, 155-59. https://doi.org/10.1126/science.aai8703. 